\title{
Management of pediatric rheumatic patients in Kazakhstan during the coronavirus disease 2019 (COVID-19) pandemic
}

\author{
Zaure Mukusheva $^{1}$ (D) $\cdot$ Maikesh Assylbekova ${ }^{1}$ (D) $\cdot$ Dimitri Poddighe $^{2}$ (D)
}

Received: 4 May 2020 / Accepted: 27 May 2020 / Published online: 8 June 2020

(c) Springer-Verlag GmbH Germany, part of Springer Nature 2020

Keywords COVID-19 $\cdot$ Diagnostic-therapeutic protocols $\cdot$ Pediatric rheumatology $\cdot$ Kazakhstan $\cdot$ Central Asia $\cdot$ Medical management

The current COVID-19 pandemic created several and important issues for the management of patients with chronic diseases. Even if children are not the population most at risk of developing severe forms of COVID-19, rheumatic diseases and the related immunosuppressive therapies are known to predispose to several infections $[1,2]$.

In general, this pandemic affected the medical management of rheumatic patients, especially in those world areas characterized by the highest incidence of COVID-19. In China, Liu et al. recommended the implementation of a remote consultation system by rheumatologists to decrease the social contacts in the hospitals, in addition to encouraging the application of all well-known hygienic and individual protection rules. As for the therapy, rheumatic patients were recommended to maintain their current medications, unless they develop COVID-19: in this case, any decision should be made according to the specific and individual patient's situation [2]. This approach was consistent with the recommendations provided by several panels of experts internationally [3].

Similar considerations were made by Favalli et al. in Italy: their rheumatic patients were told to maintain their

Dimitri Poddighe

dimitri.poddighe@nu.edu.kz

Zaure Mukusheva

zaure.mukusheva@umc.org.kz

Maikesh Assylbekova

Maykesh.Asylbekova@umc.org.kz

1 Department of Pediatric Rheumatology, National Research Center of Mother and Child Health, University Medical Center, Nur-Sultan, Kazakhstan

2 Department of Medicine, Nazarbayev University School of Medicine, Kerei-Zhanibek Str. 5/1, 010000 Nur-Sultan, Kazakhstan ongoing therapy and apply all the general rules to prevent the infections. Through an extended survey (including 530 rheumatic patients), the same authors reported only three patients infected with SARS-CoV-2, who actually developed mild clinical forms [4]. As regards pediatric rheumatic patients, the same Italian hospital in Milan, which follows up more than 2000 patients (including almost 500 children on biologic therapies) provided similar recommendations; importantly, the access to the emergency department was firmly discouraged unless really necessary. Therefore, they set up a dedicated phone number at the hospital for their patients, and both the outpatient visits and inpatient hospitalizations were limited to urgent cases or circumstances [5, 6].

A similar and even more restrictive approach has been adopted by the National Research Center for Mother and Child Health (NRCMCH), a pediatric tertiary hospital in Nur-Sultan (the capital of Kazakhstan), which includes a referral center for Pediatric Rheumatology. Our department follows more than 600 pediatric patients affected with rheumatic diseases (indicatively, Juvenile Idiopathic Arthritis: 70\%; Scleroderma: 12\%; Systemic Lupus Erythematosus: 5\%; Juvenile Dermatomyositis: 4\%; other rheumatic diseases: $9 \%$ ) treated with conventional and/or biological disease-modifying anti-rheumatic drugs, according to the international guidelines and recommendations [7, 8]. Indeed, in addition to the national state of emergency and the city lockdown, a strict quarantine was established in Nur-Sultan in mid-March, immediately after the first case of COVID-19 was detected in the country. Fortunately, here this approach resulted in a much lower number of COVID-19 cases, compared to many Western countries and China: at the moment (mid-May), less than 9,000 cases have been detected all over the country, including 34 ascertained victims only [9].

Because of the city quarantine, no one was allowed to enter or exit the city with very few exceptions: therefore, it 
was not possible for our pediatric patients (coming from all the country) to attend their scheduled specialist consultations and follow-up investigations at the NRCMCH.

Even though some children with COVID-19 have been reported in Kazakhstan (so far, $2.3 \%$ and $4.6 \%$ of total COVID-19 cases were, respectively, in the age groups 1-10 years and 11-20 years), no fatal cases were recorded among them until the first week of May [9]. Interestingly, no SARS-CoV-2 infections have been self-reported among the pediatric rheumatic patients followed at the NRCMCH by patients' families and/or peripheral hospitals, taking care of them in this period and being in close communication with the NRCMCH.

Indeed, the work of rheumatologists and, more generally, the organization of the health care in Kazakhstan have undergone some profound changes in this period. Rheumatic patients with exacerbations and those needing scheduled infusions with biological drugs had to be admitted to the local hospitals near their place of residence, even though no specialized centers were present. Accordingly, the pediatric rheumatology specialists of the NRCMCH have implemented a system of distance consultation by phone and/or video-calls to grant the appropriate therapy adjustments and provide the needed guidance for the most complex cases.

Thanks to the low incidence of COVID-19 cases in Kazakhstan so far, the most vulnerable populations, including children with rheumatic disorders, were protected from this disease. Moreover, the local health authorities have not suffered to procure the needed drugs and provide diagnostic investigations to our rheumatic patients. Even if some concerns remain for the appropriate management and followup of patients living in some remote regions of the country (where the access to well-equipped hospitals and medical care may have been more problematic), the availability of national specific guidelines for the diagnostic-therapeutic management of the main pediatric rheumatic diseases, as part of the regular health system organization in Kazakhstan, definitely contributed to maintain appropriate standards of care also in those less resourced settings. This aspect was previously emphasized by Misra et al. in their analysis on the needs and issues related to the management of rheumatic diseases in developing countries [10].

In conclusion, the less severe COVID-19 epidemiological situation in Kazakhstan so far, along with the implementation of distance consulting and the availability of national diagnostic-therapeutic disease-specific protocols even before the pandemic, have certainly lessened the burden of the negative consequences on Kazakhstani children affected with rheumatic diseases. In detail, the availability of official national protocols (based on the international guidelines) accessible to all the physicians all over the country, may be a fundamental tool for all those emergency situations in which the access to the referral and specialized medical centers is not possible, especially in developing and/or less resourced countries.

Author contributions ZM, MA and DP conceived and wrote the manuscript.

\section{Compliance with ethical standards}

Conflict of interest The authors have no conflict of interest to declare.

\section{References}

1. Parri N, Lenge M, Buonsenso D, Coronavirus Infection in Pediatric Emergency Departments (CONFIDENCE) Research Group (2020) Children with Covid-19 in Pediatric Emergency Departments in Italy. N Engl J Med. https://doi.org/10.1056/NEJMc 2007617 (Epub ahead of print)

2. Liu Y, Chang C, Lu Q (2020) Management strategies for patients with autoimmune diseases during the COVID-19 pandemic: a perspective from China. Eur J Rheumatol. https://doi.org/10.5152/ eurjrheum.2020.2056 (Epub ahead of print)

3. Misra DP, Agarwal V, Gasparyan AY, Zimba O (2020) Rheumatologists' perspective on coronavirus disease 19 (COVID-19) and potential therapeutic targets. Clin Rheumatol. https://doi. org/10.1007/s10067-020-05073-9 (Epub ahead of print)

4. Favalli EG, Ingegnoli F, Cimaz R, Caporali R (2020) What is the true incidence of COVID-19 in patients with rheumatic diseases? Ann Rheum Dis. https://doi.org/10.1136/annrheumdis-2020217615

5. Costi S, Caporali R, Cimaz R (2020) Dealing with COVID-19 in a Pediatric Rheumatology Unit in Italy. Paediatr Drugs. https:// doi.org/10.1007/s40272-020-00395-2 (Epub ahead of print)

6. Licciardi F, Giani T, Baldini L, Favalli EG, Caporali R, Cimaz R (2020) COVID-19 and what pediatric rheumatologists should know: a review from a highly affected country. Pediatr Rheumatol Online J 18(1):35

7. Poddighe D, Mukusheva Z, Dauyey K, Assylbekova M (2019) Adalimumab in the treatment of pediatric Behçet's disease: casebased review. Rheumatol Int 39(6):1107-1112

8. Poddighe D, Romano M, Gattinara M, Gerloni V (2018) Biologics for the treatment of juvenile idiopathic arthritis. Curr Med Chem 25(42):5860-5893

9. Hot Line of The Ministry of Health of The Republic of Kazakhstan. https://www.coronavirus2020.kz Accessed 16 May 2020

10. Misra DP, Sharma A, Agarwal V (2018) Guidelines for management of rheumatic diseases in developing countries from basics to real-world situation: relevance, need, and processes for development. Rheumatol Int 38(4):549-556

Publisher's Note Springer Nature remains neutral with regard to jurisdictional claims in published maps and institutional affiliations. 